\title{
The ROCK inhibitor, thiazovivin, inhibits human corneal endothelial-to-mesenchymal transition/epithelial-to-mesenchymal transition and increases ionic transporter expression
}

\author{
QIANNI WU, CHEN OUYANG, LIJIE XIE, YUNZHI LING and TING HUANG \\ State Key Laboratory of Ophthalmology, Zhongshan Ophthalmic Center, \\ Sun Yat-sen University, Guangzhou, Guangdong 510060, P.R. China
}

Received June 2, 2016; Accepted August 9, 2017

DOI: $10.3892 /$ ijmm.2017.3103

\begin{abstract}
Corneal diseases exhibit a high prevalence and are prone to cause blindness; furthermore, maintaining the morphology and ionic transporter expression in corneal endothelial cells (CECs) is crucial for treatment of these diseases. This study aimed to investigate the effects of the novel Rho associated coiled-coil containing protein kinase (ROCK) inhibitor, thiazovivin (2,4-disubstituted thiazole, TZV), on human corneal endothelial-to-mesenchymal transition/epithelial-to-mesenchymal transition (EndMT/EMT), cell morphology, junction proteins and ionic transporter expression in human CECs (HCECs) in vitro and then to clarify the mechanisms of action of TZV. In the present study, primary HCECs were cultured in vitro and passaged. The expression levels of adhesion proteins (E-cadherin and N-cadherin), the EndMT/EMT marker, $\alpha$ smooth muscle actin ( $\alpha$-SMA), the tight junction protein, Zonula occludens-1 (ZO-1), and the ionic transporter, $\mathrm{Na}^{+} / \mathrm{K}^{+}$-ATPase, were detected by immunofluorescence. The proliferative ability of the HCECs was determined by CCK- 8 assay. The mRNA expression of the EndMT/EMT-inducing gene, Snail, was examined by RT-PCR. The protein expression levels of ROCK1/2 were evaluated by western blot analysis. The HCECs were cultured with TZV at various concentrations $(2,4$, or $6 \mu \mathrm{M})$ for different periods of time ( 24 or $48 \mathrm{~h}$ ). We found that the the cell states of the HCECs co-cultured with $4 \mu \mathrm{M}$ TZV for $48 \mathrm{~h}$ reached the optimum, and corneal EndMT/EMT was inhibited, as evidenced by the significantly upregulated expression of ZO-1 and E-cadherin, and the markedly downregulated expression of $\mathrm{N}$-cadherin
\end{abstract}

Correspondence to: Dr Ting Huang, State Key Laboratory of Ophthalmology, Zhongshan Ophthalmic Center, Sun Yat-sen University, 54 Xianlie South Road, Guangzhou, Guangdong 510060, P.R. China

E-mail: thuang@vip.163.com

Key words: human corneal endothelial cells, epithelial-to-mesenchymal transition, endothelial-to-mesenchymal transition, thiazovivin, morphology, cell junctions, ionic transporter expression and $\alpha$-SMA. Furthermore, the cells exhibited a normal, tightly connected hexagonal or pentagonal morphology. Additionally, the protein expression of ROCK $1 / 2$ and the mRNA expression of Snail were significantly decreased. However, there was no significant difference between the TZV-treated and the control groups as regards HCEC proliferative ability. These findings suggested that the ROCK inhibitor, TZV $(4 \mu \mathrm{M})$, was effective in improving the morphology, cell junctions and ionic transporter expression of HCECs by inhibiting EndMT/EMT, but had no effect on HCEC proliferation.

\section{Introduction}

Corneal diseases exhibit a high prevalence and are prone to cause blindness. Normal corneal endothelial cells (CECs) are crucial for maintaining corneal transparency and clear vision. The CEC layer primarily transports nutrients in the aqueous humor to the cornea through ion channels and ion pumps. These cells also transport water molecules from the matrix to the aqueous humor to offset passive matrix swelling caused by water absorption and to maintain the relatively dehydrated state of the matrix, thus further maintaining the transparency of the cornea (1). Human corneal endothelial cells (HCECs) undergo contact inhibition due to cell junctions and the arrest of cell proliferation at the the G1 phase, thus ensuring their monolayer features and maintaining a normal corneal endothelial cell density (ECD) (2). After birth, HCECs cannot regenerate, and the loss of endothelial cells caused by injury, inflammation and eye surgery is compensated through an increase in the adjacent cell volume, a reduction in the cell density (3), or a transition into fibroblast-like cells (4), which have poor repair functions in the acute and chronic injury of CECs $(5,6)$. In the normal physiological state, with increased age, the ECD gradually decreases at a rate of 0.3-0.6\% annually, and the CEC volume also gradually increases (7). Once the corneal ECD is lower than the critical density for maintaining the physiological functions of endothelial cells $\left(400-700 / \mathrm{mm}^{2}\right)$, the cornea will undergo irreversible pathological changes, and the barrier and ionic transporter expression of CECs will be decompensated to produce corneal edema and the reduction of corneal transparency, thus inducing visual impairment. Therefore, the restoration of the normal amount, morphology and ionic 
transporter expression of CECs is of significance in the drug and surgical treatment of corneal endothelial diseases.

Penetrating keratoplasty and endothelial keratoplasty are currently the only methods for effectively treating corneal endothelial decompensation diseases. However, these surgeries have high requirements for donor corneal endothelial quality and cell number. The lack of donor corneal material suitable for corneal transplantation remains the largest limiting factor in current corneal transplantation surgery. In addition, issues such as post-operative immune rejection, endothelial dysfunction and secondary glaucoma are also common reasons for surgical failure (8). For patients at the early stage of the diseases (when corneal limbal stem cells and corneal endothelial progenitor cells still maintain their normal functions) and for patients who cannot receive corneal transplantation surgery in time, drug treatment is very important $(5,9)$. The in vitro disruption of the junctions between CECs and the application of reagents, such as growth factors in the culture environment can stimulate CEC replication (10). However, CECs obtained from culture, whether primary cells or passaged cells, often undergo fibrotic transitions with respect to cell morphology (11-14). It has been reported that endothelial-to-mesenchymal transition (EndMT) and epithelial-to-mesenchymal transition (EMT) are major factors limiting the production of the CEC layer graft in tissue engineering, which can not only result in the dissociation of cell junctions, the lack of apical-basal polarity, changes in cell morphology and an increase in cell motility, but can also lead to the re-organization of the Rho guanosine triphosphatase family-dependent actin cytoskeleton, promoting the production of extracellular matrix proteins and changing gene expression $(15,16)$. Therefore, in cultured CECs, EndMT and EMT, are the two most important factors which can cause CECs to lose their normal cell morphology and can induce cell fibrosis (17).

Thiazovivin (2,4-disubstituted thiazole, TZV) is a novel Rho associated coiled-coil containing protein kinase (ROCK) inhibitor. Currently, in vitro studies on ROCK inhibitors primarily focus on the effect of selective inhibitors, such as Y-27632 and Y-39983 on the proliferation of cynomolgus monkey and rabbit CECs and cell scratch tests (18). Studies on TZV are restricted to embryonic stem cells, adult endometrial stromal cells and neurons (19-21). In this study, we cultured primary HCECs and then passaged them. In addition, the effects of TZV on EndMT/EMT, cell morphology, junction proteins and ionic transporter expression in HCECs in vitro were investigated, and the underlying mechanisms were also examined.

\section{Materials and methods}

\section{Materials}

Human corneal tissue material. All procedures in this study conformed to the Declaration of Helsinki developed by the World Medical Association (WMA) and the ethics principles of the International Ethical Guidelines for biomedical research involving human subjects developed by the Council for International Organizations of Medical Sciences (CIOMS). This study was approved by the Ethics Committee of Zhongshan Ophthalmic Center, Sun Yat-sen University. Written informed consent was obtained from all donors prior to obtaining the samples. The human corneal tissues used in this study were corneal limbi left from corneal transplantation surgery and were all from the Eye Bank of Guangdong Province. After receiving the donor corneal materials, the Eye Bank performed screening for health, past disease history and genetic disease history of the corneal donors to ensure that the corneal tissues were in excellent condition and suitable to be used as the graft material for corneal transplantation surgery.

Experimental materials. FNC coating mix was purchased from Athena Environmental Sciences, Inc. (Baltimore, MD, USA); Opti-MEM-I (1X) reduced serum medium, pen strep (penicillin streptomycin), $0.25 \%$ trypsin-EDTA (1X), PBS (10X), bovine serum albumin (BSA), Australian fetal bovine serum and goat serum were all purchased from Gibco (Mountain View, CA, USA). Petri dishes (35 mm) with lids, culture plates, chamber culture plates, DAPI, Super ECL chemiluminescent solution, and collagenase IV were all purchased from Sigma (St. Louis, MO, USA). N-cadherin (D4R1N) XP ${ }^{\circledR}$ rabbit monoclonal antibody (mAb) (\#13116), E-cadherin (24E10) (24E10) rabbit mAb (Alexa Fluor ${ }^{\circledR} 488$ conjugate, \#3199), $\mathrm{Na}^{+} / \mathrm{K}^{+}$-ATPase $\alpha 1$ (D4Y7E) rabbit mAb (\#23565), neuronspecific enolase (NSE) antibody, anti-rabbit IgG (H+L), F(ab') fragment (Alexa Fluor ${ }^{\circledR} 488$ conjugate; \#4412), anti-rabbit IgG, HRP-linked antibody (\#7074) and GAPDH (14C10) rabbit $\mathrm{mAb}$ (\#2118) were all purchased from Cell Signaling Technology, Inc. (Danvers, MA, USA). Purified mouse anti-human Zonula occludens-1 (ZO-1) antibody (\#610967) was purchased from BD Biosciences (Franklin Lakes, NJ, USA). Anti- $\alpha$ smooth muscle actin antibody ( $\alpha$-SMA; ab5694) and goat anti-mouse IgG H\&L (Alexa Fluor ${ }^{\circledR} 594$; ab150120) were purchased from Abcam (Cambridge, UK). Anti-ROCK-1/2 antibody (\#07-1458) was purchased from Merck Millipore (Billerica, MA, USA). Epidermal growth factor (EGF; E9644), calcium chloride (C5670), chondroitin sulfate A (C9819) and L-ascorbic acid (A4403) were all purchased from Sigma. TZV and DMSO were purchased from Selleck Chemicals (Houston, TX, USA). Y-27632 (CalBiochem, San Diego, CA, USA), qPCR reagent $\mathrm{SYBR}^{\circledR}$ Premix Ex Taq $^{\mathrm{TM}}$, RNA extraction reagent RNAiso Plus, reverse transcriptase reagent PrimeScript ${ }^{\mathrm{TM}}$ RT Master Mix, and the CCK-8 reagent kit were purchased from Takara Bio (Dalian, China).

\section{Methods}

Cell culture. The corneal materials used in this study were from corneal limbi left from corneal transplantation surgery $(n=6)$. The ECD range of the corneal graft materials was $1,500-2,000 / \mathrm{mm}^{2}$. The age of the corneal donors was 22-48 years. Descemet's membrane and the endothelial layer were isolated under a dissection microscope and immersed in $4 \%$ type IV collagenase for 15-20 min. After complete culture medium was added and centrifuged 3 times $(1,500 \mathrm{rpm} / \mathrm{min}$, $5 \mathrm{~min}$ ), Descemet's membrane and CECs were inoculated together onto FNC-coated 35-mm Petri dishes with lids, 4-,8- and 6-well chamber culture plates and placed in a $37^{\circ} \mathrm{C}$, $5 \% \mathrm{CO}_{2}$ incubator. After 15-20 days, when the primary HCECs effectively covered the bottom of the Petri dish and the confluence reached approximately $80 \%$, passage and other subsequent experiments were performed. The complete culture medium contained Opti-MEM-I, 8\% FBS, 1\%o Pen/Strep (Gibco), $5 \mathrm{ng} / \mathrm{ml}$ EGF (Sigma), $20 \mu \mathrm{g} / \mathrm{ml}$ ascorbic acid, $200 \mathrm{mg} / \mathrm{l}$ calcium chloride (Sigma) and $0.08 \%$ chondroitin sulfate A. 
Immunofluorescence staining. The samples were fixed in $4 \%$ paraformaldehyde for $30 \mathrm{~min}$ and blocked in $1 \% \mathrm{BSA}$ or goat serum for $1 \mathrm{~h}$. The samples were washed with $1 \mathrm{X}$ PBS 3 times for $5 \mathrm{~min}$ each on a rotary destaining shaker at $50 \mathrm{rpm}$. The primary antibodies (NSE antibody, N-cadherin antibody, E-cadherin antibody, ZO-1 antibody, $\alpha$-SMA antibody, $\mathrm{Na}^{+} / \mathrm{K}^{+}$-ATPase antibody and ROCK antibody) were diluted in primary antibody dilution solution according to the ratio in the instruction manual. The samples were incubated with primary antibodies at $4^{\circ} \mathrm{C}$ for $12 \mathrm{~h}$. The samples were washed with 1X PBS 3 times for $5 \mathrm{~min}$ each on a rotary destaining shaker at $50 \mathrm{rpm}$. Based on the sources of the primary antibodies, corresponding secondary antibodies were selected. Secondary antibodies were diluted using the secondary antibody dilution solution (0.01 M PBS containing 1\% BSA and $0.3 \%$ Triton $\mathrm{X}-100, \mathrm{pH} 7.4$ ) according to the instruction manual. The samples were incubated with secondary antibodies at $4^{\circ} \mathrm{C}$ for $4 \mathrm{~h}$. The samples were washed with $1 \mathrm{X}$ PBS 3 times for $5 \mathrm{~min}$ each on a rotary destaining shaker at $50 \mathrm{rpm}$. The samples were then incubated in $0.5 \mu \mathrm{g} / \mathrm{ml}$ DAPI at room temperature for $10 \mathrm{~min}$, washed with $1 \mathrm{X}$ PBS, and finally mounted in an anti-fluorescence quenching agent. The results were observed using an automatic upright fluorescence microscope (Imager Z1; Zeiss, Jena, Germany).

Determination of proliferative ability. The cell suspension was inoculated onto 96 -well plates $(100 \mu \mathrm{l} /$ well). Culture plates were placed in an incubator for pre-culture $\left(37^{\circ} \mathrm{C}, 5 \% \mathrm{CO}_{2}\right)$. Approximately $10 \mu \mathrm{l}$ of CCK-8 solution was added to each well. The culture plates were placed in an incubator for 1-4 h. The absorbance at $450 \mathrm{~nm}$ was measured using a microplate reader (Synergy H1, Hybrid Reader; BioTek, Winooski, VT, USA).

Western blot analysis. Proteins were extracted, and protein concentrations were determined using a BCA reagent kit. All samples were diluted to the same concentration using RIPA buffer; 4X SDS sample loading buffer was added, and the samples were boiled for $5 \mathrm{~min}$, mixed thoroughly and centrifuged. The samples were loaded onto a $10 \%$ sodium dodecyl sulfate polyacrylamide gel for electrophoresis. After electrophoresis was completed, the proteins were transferred onto a PVDF membrane and blocked in 5\% non-fat milk at room temperature for $1 \mathrm{~h}$ on a shaker with stable shaking. The diluted primary antibody (ROCK) was added; the dilution was 1:1,000. The diluted rabbit-anti GADPH antibody was added as an internal control at a 1:2,000 dilution. The membrane was incubated overnight at $4^{\circ} \mathrm{C}$ on a shaker. After washing in $1 \mathrm{X}$ TBST for $10 \mathrm{~min} 3$ times, the membrane was placed in the corresponding secondary antibodies diluted in 5\% non-fat milk (secondary antibody concentration 1:5,000) and incubated at room temperature for $1 \mathrm{~h}$. Following incubation with secondary antibodies, the membrane was washed in $1 \mathrm{X}$ TBST for $10 \mathrm{~min}$ 3 times. Solution A and B in super ECL were mixed at equal volumes; after mixing thoroughly, the solution was added onto the membrane. The membrane was exposed and fixed, and the X-ray film was scanned using a scanner (5200 automatic chemiluminescence image analysis system; Tanon, Shanghai, China).

Reverse transcription-quantitative PCR (RT-qPCR). Total RNA from the cultured cells was first extracted using TriPure Isolation reagent (Invitrogen Life Technologies, Carlsbad, CA, USA). The RNA concentration and purity were detected using UV spectroscopy (Thermo Fisher Scientific, Inc., Waltham,
MA, USA). The RNA (1 $\mu \mathrm{g})$ was reverse transcribed into cDNA using the Transcriptor First Strand cDNA Synthesis kit (Roche Applied Science, Penzberg, Germany). PCR amplification was performed using a Light Cycler instrument and the Light Cycler Fast Start DNA Master SYBR-Green 1 kit (Roche Applied Science) according to the manufacturer's instructions. Finally, gene expression was quantified relatively by ABI Prism 7700 Sequence Detection system. The primers for Snail were forward, CCACACTGGCGAGAAG and reverse, AGAAGGTCCGAGCACAC; the primers for GADPH were forward, GCACCGTCAAGGCTGAGAAC and reverse, TGGTGAAGACGCCAGTGGA. GAPDH was used as an internal control, and the relative expression level of Snail was calculated using the $2^{-\Delta \Delta \mathrm{Ct}}$ method.

Cell treatment. Primary and passaged HCECs were cultured, and the cells were identified by observing the cell morphology under a light microscope and through NSE immunofluorescence staining experiments. When the confluence of primary cells was close to $70-80 \%, 0.25 \%$ trypsin-EDTA (1X) was added for digestion. Following centrifugation, the cells were sub-cultured (1-3 generations). Primary and passaged HCECs were cultured onto 8-well chamber culture plates. When the cell confluence reached approximately $80 \%$, various concentrations of TZV $(2,4$ and $6 \mu \mathrm{M}), 0.6 \%$ DMSO, or $10 \mu \mathrm{M}$ Y-27632 were added. After 24 and $48 \mathrm{~h}$, the cell morphology was observed (10 $\mu \mathrm{M}$ Y-27632 was detected after $48 \mathrm{~h})$, and the expression of E-cadherin, $\mathrm{N}$-cadherin, $\alpha$-SMA, ZO-1, and $\mathrm{Na}^{+} / \mathrm{K}^{+}$-ATPase were evaluated by immunofluorescence staining experiments. The expression of ROCK was detected by western blot analysis, and the mRNA expression of Snail was detected by RT-PCR.

Statistical analysis. All data are presented as the means \pm SD and analyzed using SPSS 13.0 software. Comparisons between groups were processed by one-way analysis of variance. A value of $\mathrm{P}<0.05$ was considered to indicate a statistically significant difference.

\section{Results}

Identification of HCECs. Primary and subcultured CECs were identified by observing the cell morphology using a light microscope and immunofluorescence staining of the CEC marker NSE. The results are presented in Fig. 1. Under a light microscope, the cells exhibited a regular hexagonal or polygonal shape, the size was homogeneous and the arrangement was dense. In addition, the cells were homogeneously stained with the NSE antibody, and the hexagonal morphology of the cells was clear. These results revealed that these cells displayed the characteristics of HCECs.

Screening of the optimal drug concentration of TZV. Fig 2 presents the effect of TZV on primary HCEC adherens junction proteins (E-cadherin and N-cadherin), the EndMT marker, $\alpha$-SMA, a tight junction protein (ZO-1) and an ionic transporter expression protein $\left(\mathrm{Na}^{+} / \mathrm{K}^{+}\right.$-ATPase). The immunofluorescence results of E-cadherin (Fig. 2A) demonstrated that the cell density of the HCECs without the addition of TZV (0.6\% DMSO control) was relatively lower than that following the addition of TZV. Furthermore, the 

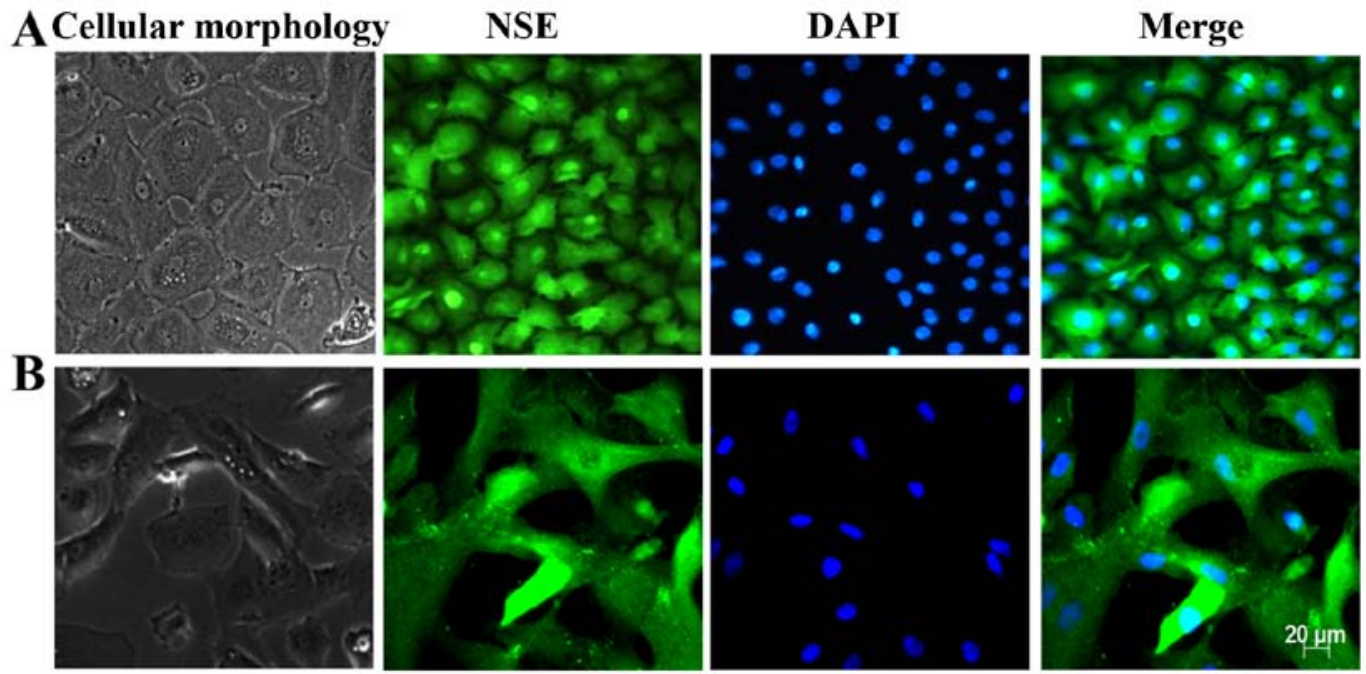

Figure 1. Light optical microscopy and immunofluorescence staining of neuron-specific enolase (NSE) in human corneal endothelial cells (HCECs), $\mathrm{n}=3$. (A) Primary cultured cells; (B) subcultured cells, (magnification x200).

fluorescence of E-cadherin was weaker, the cell morphology exhibited round or polygonal shapes, and there were no tight junctions between most cells. However, following the addition of TZV, the fluorescence expression increased. When the drug concentration was $2 \mu \mathrm{M}$, the cell number increased compared with that in the DMSO control group; however, the changes in morphology were not obvious. When the drug concentration increased to $6 \mu \mathrm{M}$, the cell volume increased, and some cells exhibited a long spindle shape and a fibroblast-like phenotype. Following the addition of $4 \mu \mathrm{M} \mathrm{TZV}$ for $24 \mathrm{~h}$, the fluorescence expression of E-cadherin was stronger, and the cells exhibited a tightly connected hexagonal or pentagonal morphology. N-cadherin was highly expressed in the HCECs and localized to the cell boundary, which could stabilize the junction between cells to maintain the normal hexagonally shaped HCEC cell structure. Thus, it appears that among the two adherens junction protein in HCECs, $\mathrm{N}$-cadherin plays a more important role than E-cadherin (6). When $4 \mu \mathrm{M}$ TZV and HCECs were co-cultured for $24 \mathrm{~h}$, compared with the DMSO group and other drug concentration groups, the fluorescence expression of $\mathrm{N}$-cadherin and $\alpha$-SMA was weaker, the HCEC volume was smaller, the cell junction was dense and clear, and the cell morphology exhibited hexagonal or pentagonal shapes. When the drug concentration was $2 \mu \mathrm{M}$, compared with the DMSO group, the changes in cell morphology and cell number were not obvious, and cell junctions were not observed. When the TZV concentration increased to $6 \mu \mathrm{M}$, the cell volume significantly increased; most cells exhibited a long spindle shape and showed a tendency toward fibrosis; furthermore, the N-cadherin and $\alpha$-SMA expression increased, paralleling the trend of E-cadherin expression. After $24 \mathrm{~h}$ of $4 \mu \mathrm{M}$ TZV treatment, immunofluorescence analysis of ZO-1 expression revealed that the cell density increased, the cell morphology was hexagonal or pentagonal shape, the ZO-1 expression level was higher, and the cell junctions were denser and clearer than those in the other control groups (Fig. 2B). When the drug concentration continuously increased, the cells exhibited spindle-shape changes. In addition, Fig. $2 \mathrm{C}$ presents the expression of $\mathrm{Na}^{+} / \mathrm{K}^{+}$-ATPase in the HCECs following treatment with various concentrations of TZV. As shown in Fig. 2C, following treatment with gradient concentrations of TZV at 2, 4 and $6 \mu \mathrm{M}$ in the HCECs for $24 \mathrm{~h}$, the fluorescence expression of $\mathrm{Na}^{+} / \mathrm{K}^{+}$-ATPase significantly increased when $\mathrm{TZV}$ was at $4 \mu \mathrm{M}$. The above-mentioned results indicated that $4 \mu \mathrm{M}$ TZV had the optimal effects on cell morphology, cell junction, cell density and pump junction in primary HCECs.

Fig. 3 presents the immunofluorescence results of E-cadherin, $\mathrm{N}$-cadherin, $\alpha$-SMA, ZO-1, and $\mathrm{Na}^{+} / \mathrm{K}^{+}$-ATPase in passaged HCECs. Compared with the primary HCECs, most of the passaged HCECs were spindle-shaped or polygonal-shaped, with no or few tight junctions between the cells. However, when $4 \mu \mathrm{M}$ TZV and passaged HCECs were co-cultured for $24 \mathrm{~h}$, the cell volume decreased, the morphology was close to a round or polygonal shape, the junctions between the cells increased, and the cell ionic transporter expression was at the optimal status compared with the findings in the other control groups.

Therefore, these results revealed that compared with the other concentrations of TZV ( 2 and $6 \mu \mathrm{M}$ ), with co-culture of $4 \mu \mathrm{M} \mathrm{TZV}$ and HCECs, the state of HCECs was maintained at the optimum.

Screening of the optimal action time of TZV. Fig. 4 presents the immunofluorescence results of adherens junction proteins (E-cadherin and $\mathrm{N}$-cadherin), the EndMT marker, $\alpha$-SMA, a ZO-1 and an ionic transporter expression protein $\left(\mathrm{Na}^{+} / \mathrm{K}^{+}\right.$-ATPase) after the HCECs were treated with the optimal drug concentration of TZV $(4 \mu \mathrm{M})$ for different periods of time ( 24 and $48 \mathrm{~h}$ ). Following treatment with $4 \mu \mathrm{M}$ TZV for $24 \mathrm{~h}$, the HCECs were tightly and regularly arranged, the cell junctions were clear, and the cell morphology exhibited an even hexagonal arrangement. With the extension of time, following treatment with TZV for $48 \mathrm{~h}$, the morphology of the HCECs improved further. Fig. 5 presents the effects of $4 \mu \mathrm{M}$ TZV at different periods of time ( 24 and $48 \mathrm{~h}$ ) on the expression of E-cadherin, $\mathrm{N}$-cadherin, $\alpha$-SMA, ZO- 1 and $\mathrm{Na}^{+} / \mathrm{K}^{+}$-ATPase in the passaged HCECs. Similarly, following treatmetn with TZV for $48 \mathrm{~h}$, the HCECs reached the optimal state. Therefore, 
A

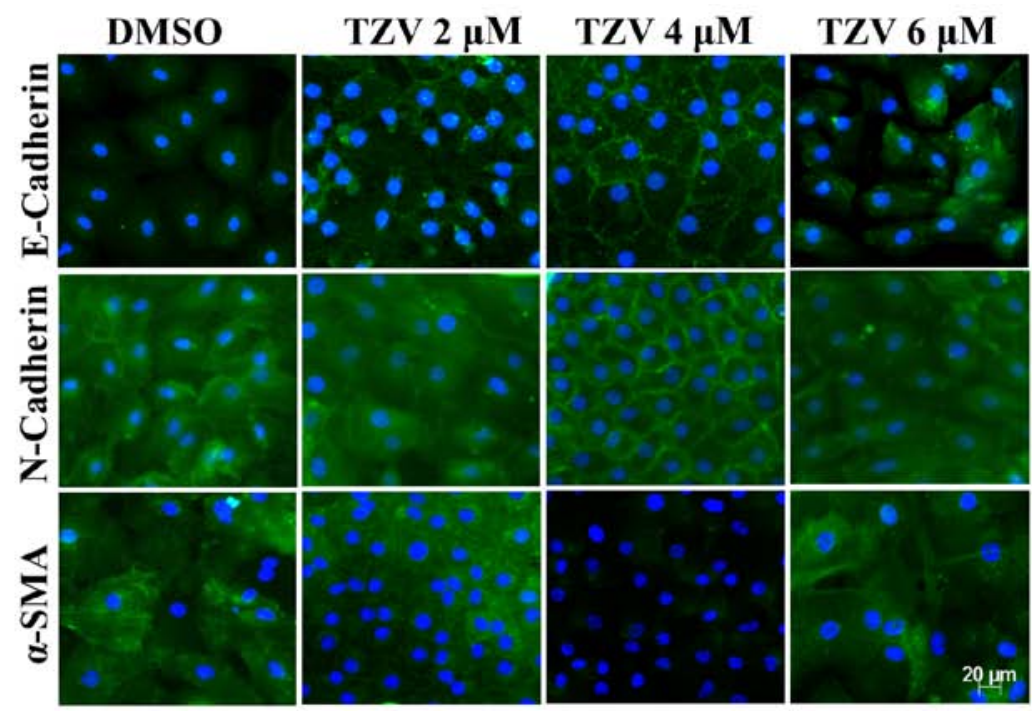

B
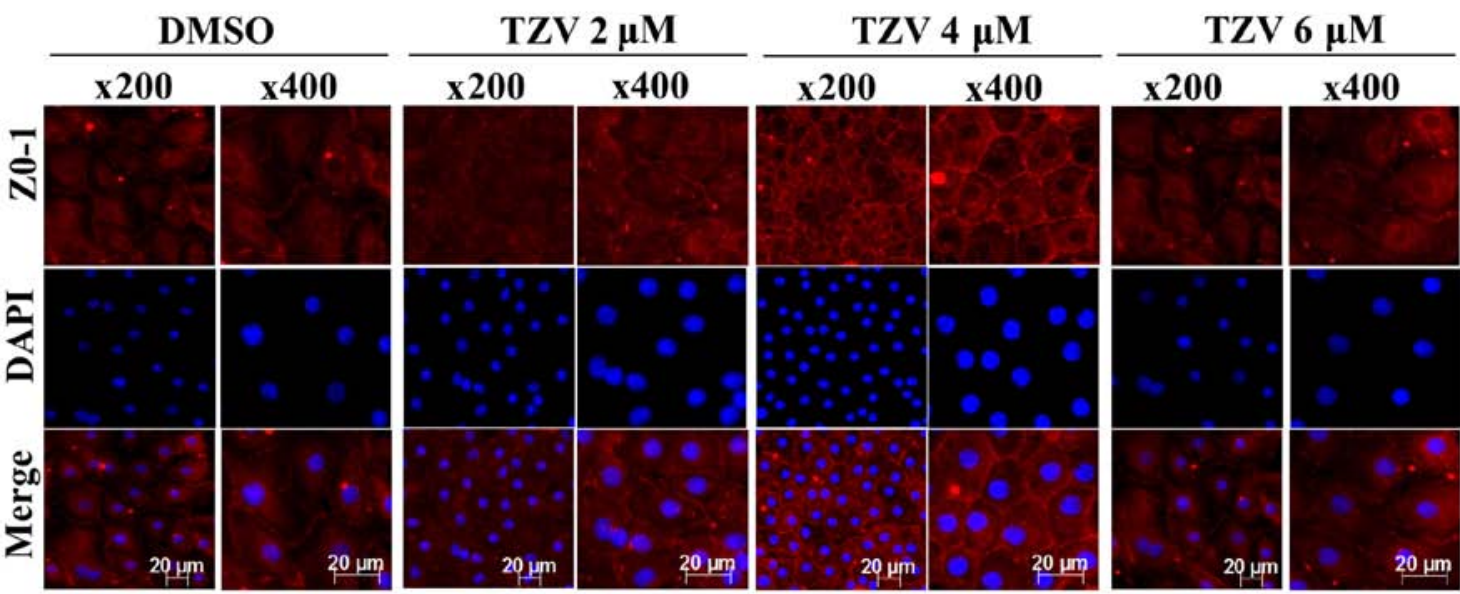

C
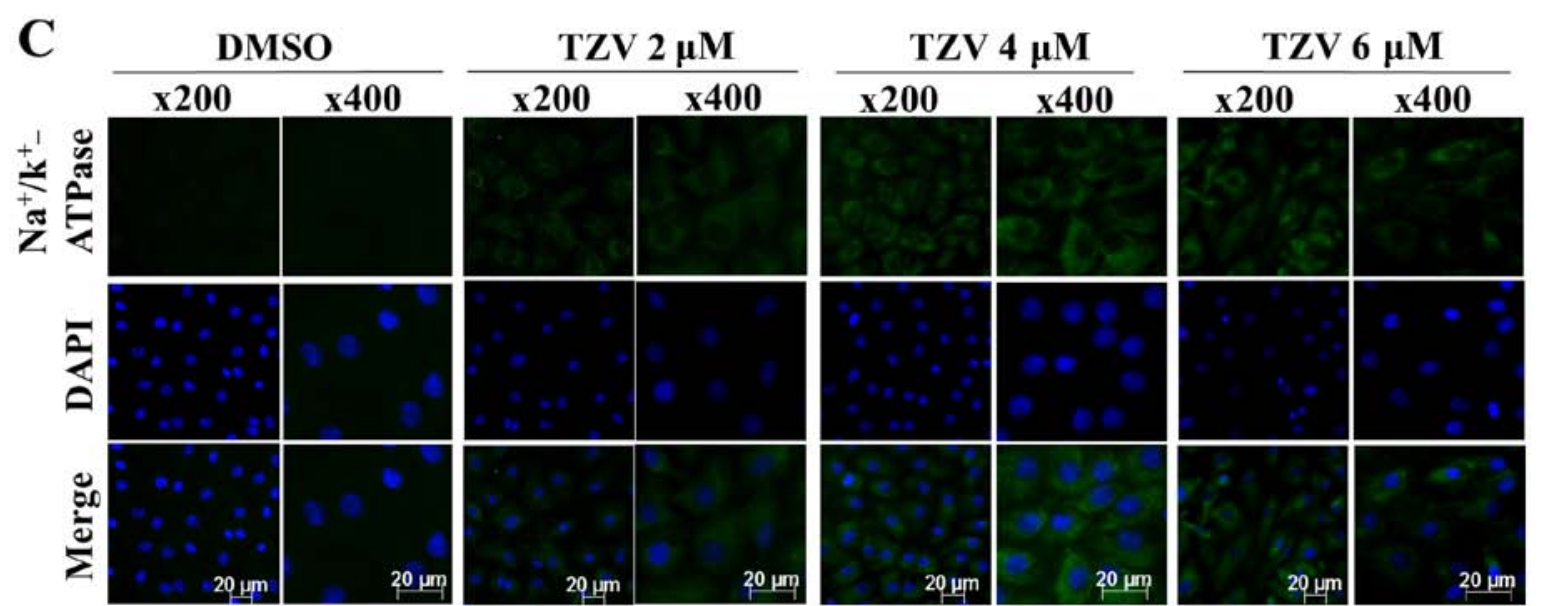

Figure 2. Function-related protein expression in primary HCECs cultured with various concentrations of TZV for $24 \mathrm{~h}, \mathrm{n}=3$. (A) E-cadherin, N-cadherin, and $\alpha$-SMA immunostaining (magnification, x200); (B) ZO-1 immunostaining; (C) $\mathrm{Na}^{+} / \mathrm{k}^{+}$-ATPase immunostaining. DMSO served as the negative control, and the concentration was $0.6 \%$. HCECs, human corneal endothelial cells; TZV, thiazovivin.

the above-mentioned results indicate that for junction proteins and ionic transporter expression, the optimal effective action time of TZV on primary and passaged HCECs was $48 \mathrm{~h}$.

Effects of TZV on HCEC proliferation. Fig. 6 presents the effects of TZV on the proliferation of HCECs. As shown in Fig. 6 , we found that compared with the DMSO group and the positive control (Y-27632 group), there was no significant difference in the TZV-treated group. The results from CCK-8 assay revealed that TZV had no effect on HCEC proliferation.

Effects of TZV on ROCK. ROCK belongs to the serine/threonine protein kinase family. ROCK is primarily distributed in the cytoplasm and functions to adjust the motility of cytoskeleton 
A

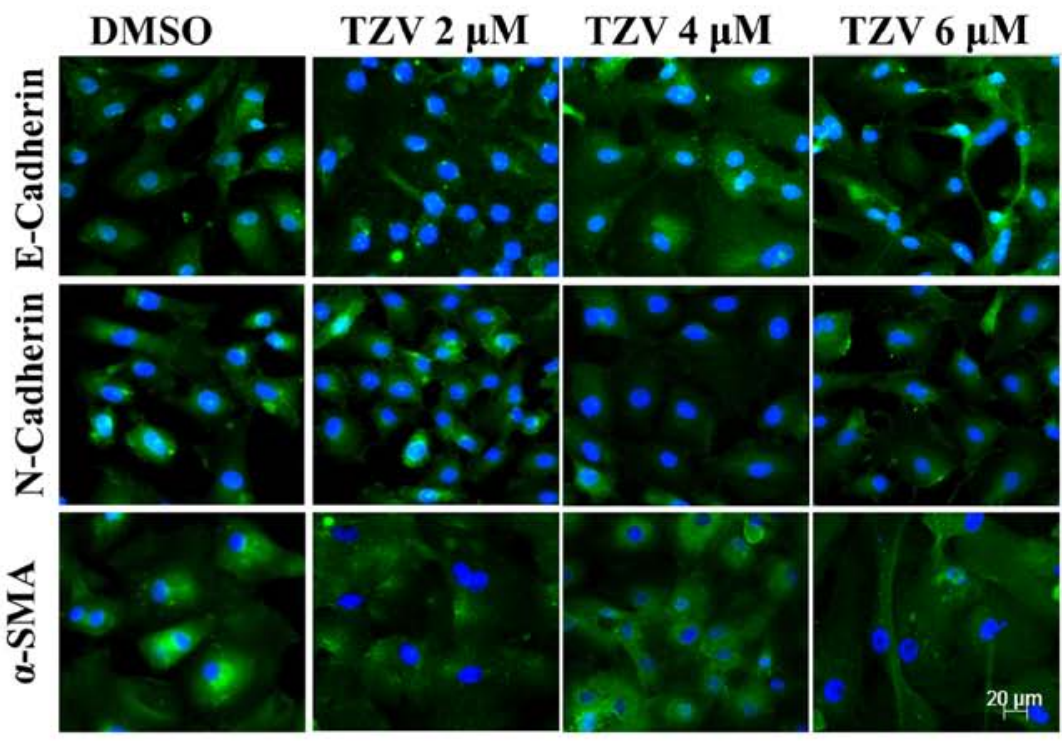

B

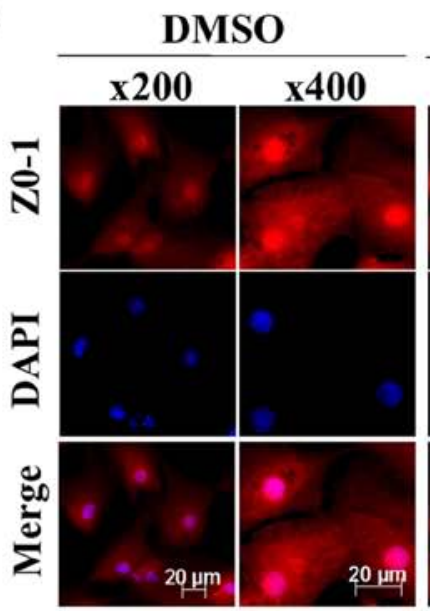

\begin{tabular}{cc} 
TZV $2 \mu \mathrm{M}$ \\
\hline $\mathbf{x} 200$ & $\mathbf{x} 400$
\end{tabular}
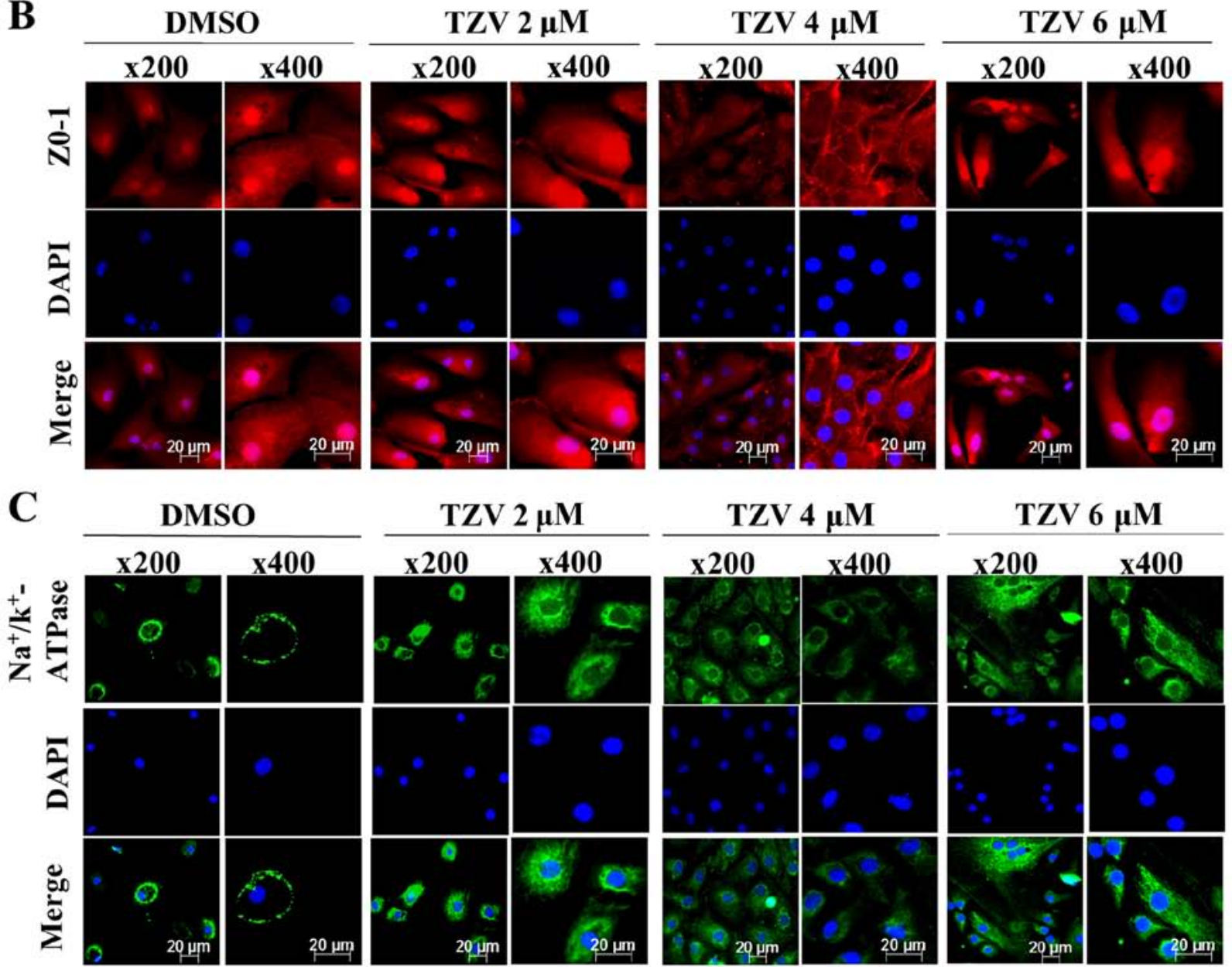

Figure 3. Function-related protein expression in passaged HCECs cultured with various concentrations of TZV for 24 h, n=3. (A) E-cadherin, N-cadherin and $\alpha$-SMA immunostaining (magnification, $\mathrm{x} 200$ ); (B) ZO-1 immunostaining; (C) $\mathrm{Na}^{+} / \mathrm{k}^{+}$-ATPase immunostaining. DMSO served as the negative control, and the concentration was $0.6 \%$. HCECs, human corneal endothelial cells; TZV, thiazovivin.

components. The high expression and over-activation of ROCK can cause disease development (22). In this study, the effects of TZV on ROCK expression were observed using western blot analysis. Fig. 7 presents the protein expression of ROCK $1 / 2$ in the HCECs cultured with $4 \mu \mathrm{m}$ TZV for different periods of time. The results revealed that after the HCECs were treated with TZV for 24 and $48 \mathrm{~h}$, the expression of ROCK1/2 significantly decreased. In addition, we found that TZV treatment for $48 \mathrm{~h}$ led to a much lower expression of ROCK1/2, compared with TZV treatment for $24 \mathrm{~h}$. These 
A

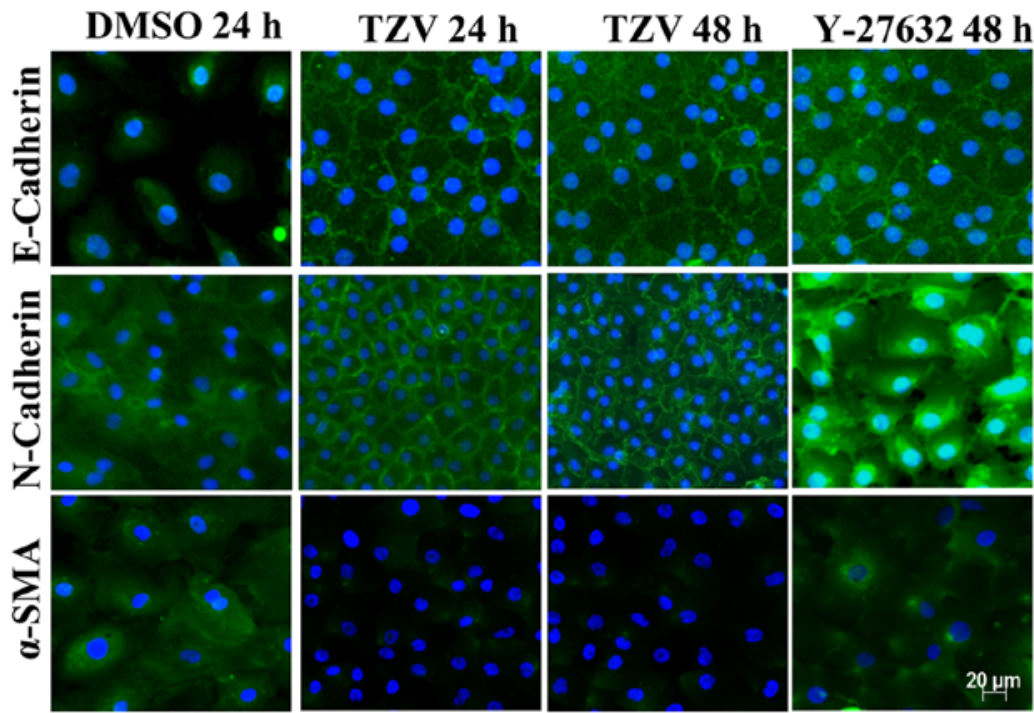

B
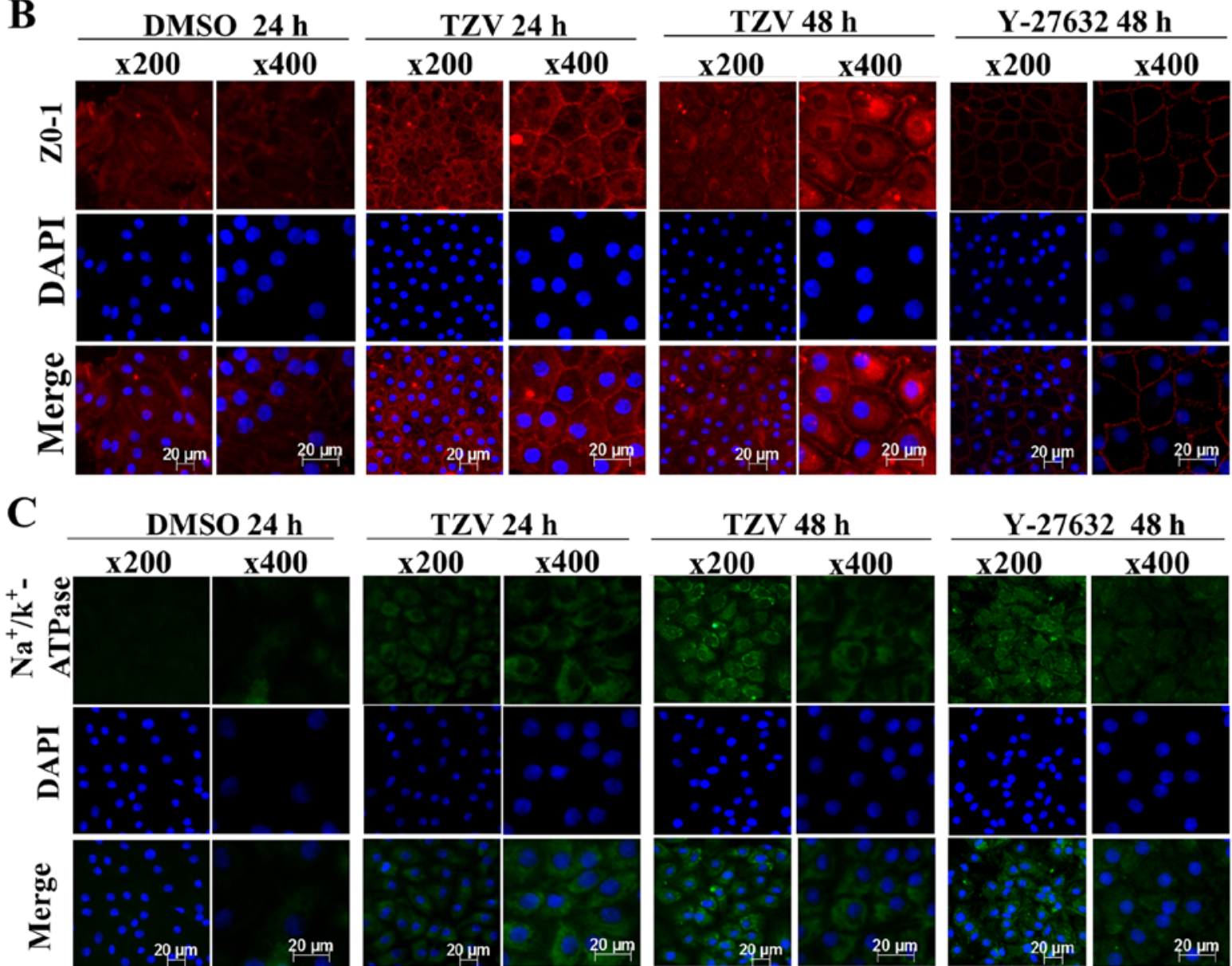

Figure 4. Function-related protein expression in primary HCECs cultured with $4 \mu \mathrm{M}$ TZV for different periods of time, $\mathrm{n}=3$. (A) E-cadherin, N-cadherin and $\alpha$-SMA immunostaining (magnification, x200); (B) ZO-1 immunostaining; (C) $\mathrm{Na}^{+} / \mathrm{k}^{+}$-ATPase immunostaining. DMSO served as the negative control, and the concentration was $0.6 \%$; Y-27632 served as the positive control, and the concentration was $10 \mu \mathrm{M}$. HCECs, human corneal endothelial cells; TZV, thiazovivin.

results indicate that TZV indeed effectively inhibited ROCK protein expression in HCECs.

TZV inhibits EndMT/EMT in HCECs. Fig. 8 presents the effects of TZV on the mRNA expression level of the EndMT/EMT regulatory gene, Snail. The expression of the EMT-inducing factor, Snail, can cause the disruption of cell junctions, which is an important marker for transition into a mesenchymal cell-like status $(23,24)$. After the primary and passaged HCECs were treated with $4 \mu \mathrm{M}$ TZV for 24 and $48 \mathrm{~h}$, the expression level of Snail was detected by RT-PCR. Compared with the DMSO group, $4 \mu \mathrm{M}$ TZV significantly inhibited Snail expression after primary culture of the HCECs for $48 \mathrm{~h}(\mathrm{P}<0.001)$, and no significant difference was observed after the HCECs were cultured 
A

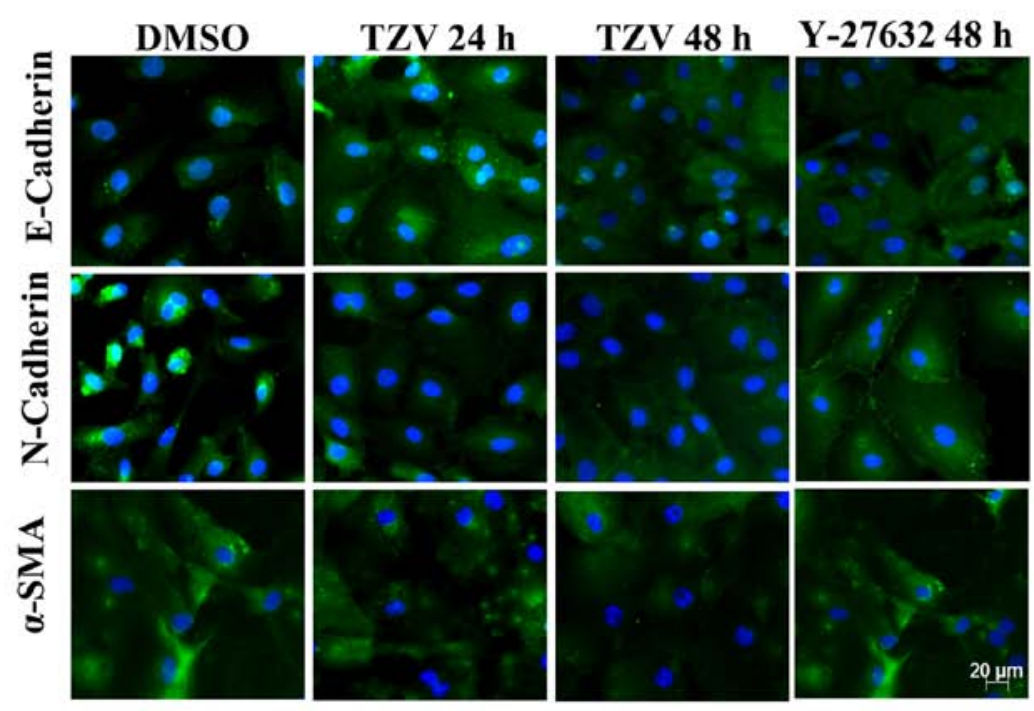

B
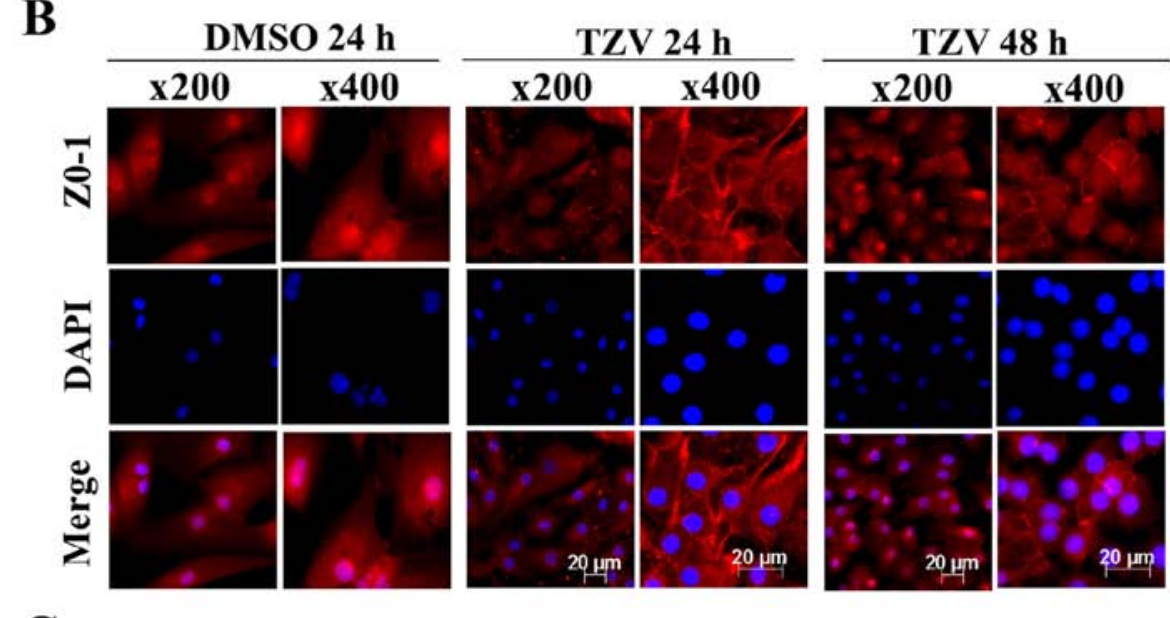

Y-27632 $48 \mathrm{~h}$

C
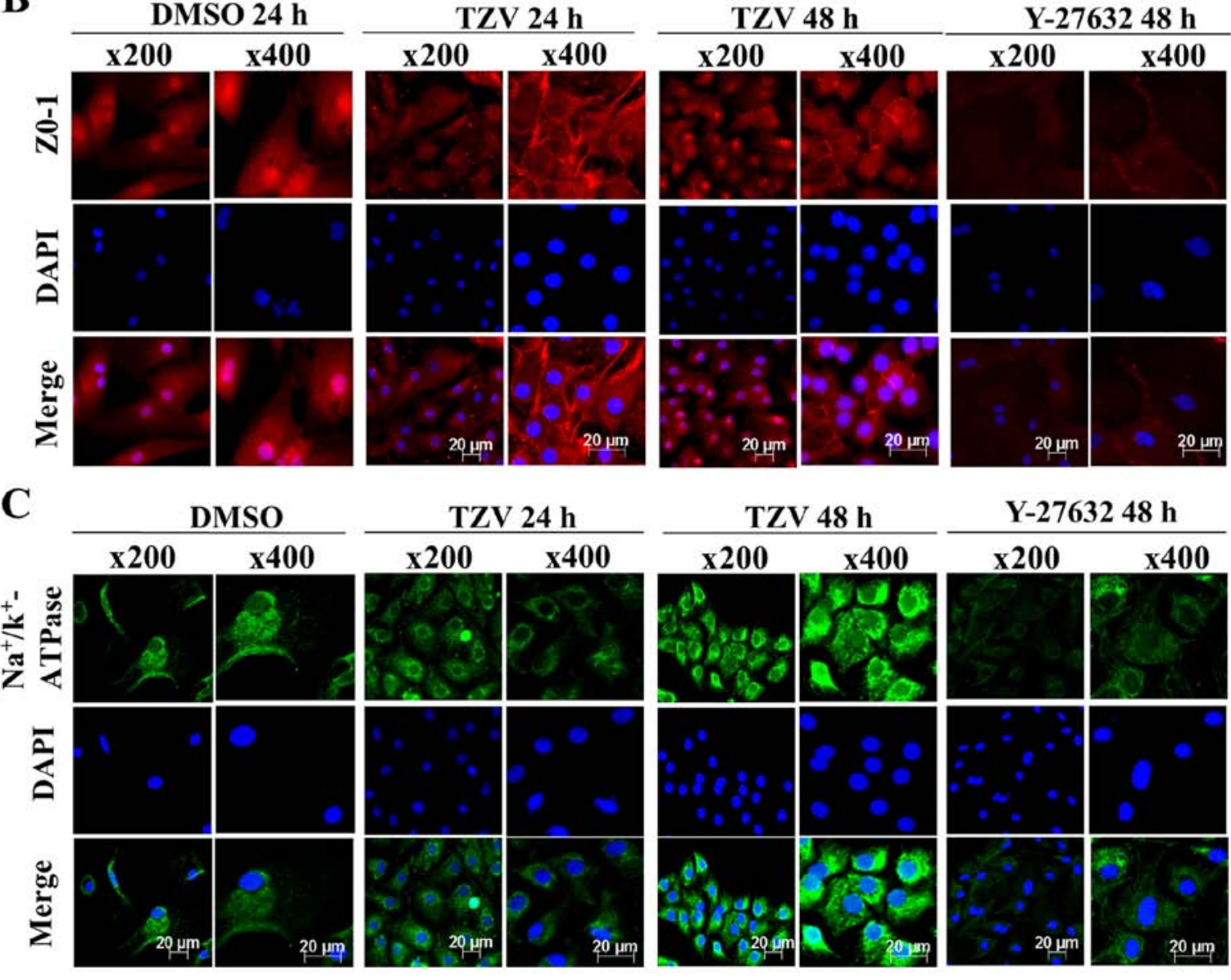

Figure 5. Function-related protein expression in passaged HCECs cultured with $4 \mu \mathrm{M}$ TZV for different periods, $\mathrm{n}=3$. (A) E-cadherin, N-cadherin, and $\alpha$-SMA immunostaining (magnification x200); (B) ZO-1 immunostaining; (C) $\mathrm{Na}^{+} / \mathrm{k}^{+}$-ATPase immunostaining. DMSO served as the negative control, and the concentration was $0.6 \%$; Y-27632 served as the positive control, and the concentration was $10 \mu \mathrm{M}$. HCECs, human corneal endothelial cells; TZV, thiazovivin.

with TZV for $24 \mathrm{~h}$ (Fig. 8A). As shown in Fig. 8B, we found that the passaged HCECs cultured with $4 \mu \mathrm{M}$ TZV for 24 and $48 \mathrm{~h}$ exhibited significant differences compared with the control.

\section{Discussion}

The morphological integrity and healthy function of HCECs are clinical indicators of corneal function. To date, at least to the best of our knowledge, there are no reports on the effects of the ROCK inhibitor, TZV, on the cell morphology and ionic transporter expression in HCECs. ROCK can regulate the synthesis, degradation, motility and contraction of cytoskeleton proteins and participates in the basic life cycle of cells, thus playing important roles in cell junctions, morphology and motility $(25,26)$. Specifically, ROCK can regulate actin stress fiber assembly and cell contraction via the phosphorylation 


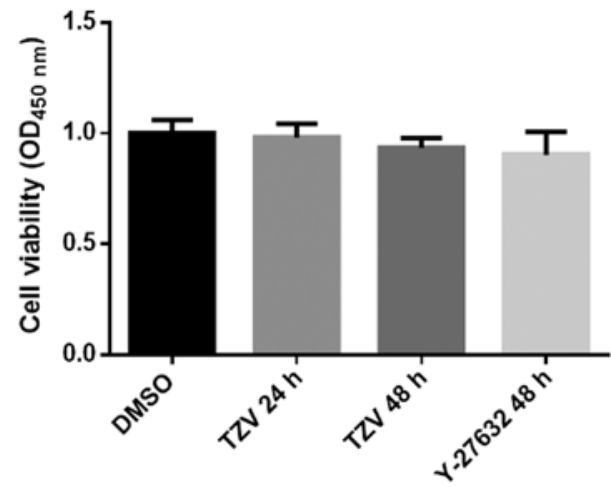

Figure 6. Cell viability of HCECs cultured with $4 \mu \mathrm{M}$ TZV for different periods of time, $n=3$. HCECs, human corneal endothelial cells; TZV, thiazovivin.

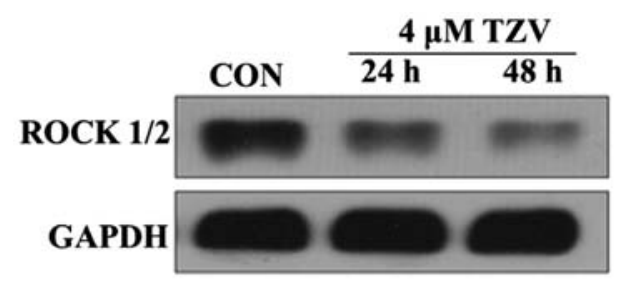

Figure 7. Protein expression of ROCK1/2 in HCECs cultured with $4 \mu \mathrm{M}$ TZV for different periods of time, $n=3$. HCECs, human corneal endothelial cells; $\mathrm{TZV}$, thiazovivin.
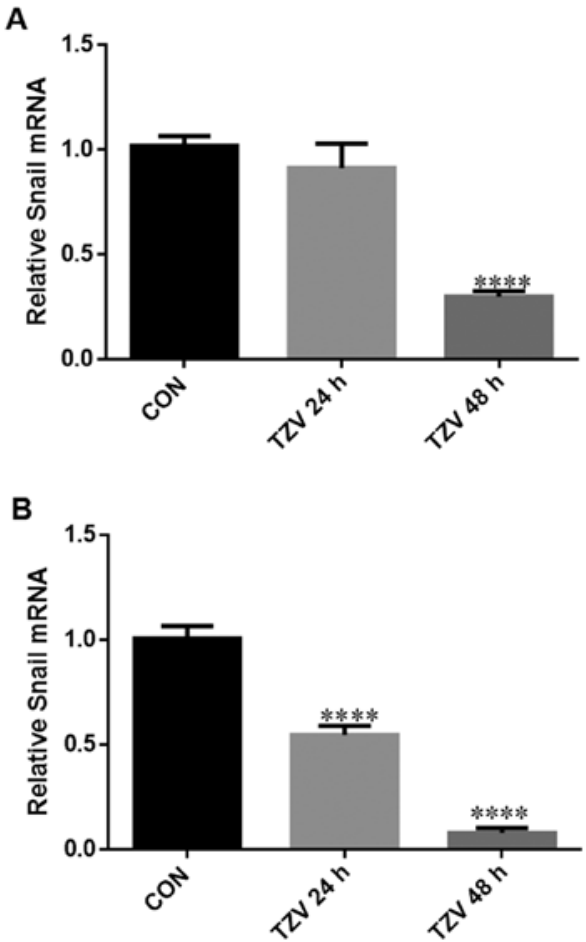

Figure 8. mRNA expression of Snail in HCECs cultured with $4 \mu \mathrm{m}$ TZV for different periods of time. (A) Primary cells; (B) subcultured cells; HCECs, human corneal endothelial cells; TZV, thiazovivin. Data are expressed as the means \pm SD of 3 experiments, ${ }^{* * *} \mathrm{P}<0.001 ;{ }^{* * * *} \mathrm{P}<0.0001$.

of its downstream molecules, myosin light chain, myosin light chain phosphatase, ezrin-radixin-moesin proteins and LIM kinase (27), and can form local cell junctions via the phosphorylation of $\mathrm{Na}^{+} / \mathrm{H}^{+}$exchanger 1 (NHE1) (28). Furthermore, in addition to regulating cytoskeleton remodeling and cell migration, ROCK signaling pathways are also closely associated with gene transcription, the promotion of cell cycle progression of G1 cells, and cell apoptosis $(29,30)$. Currently, ROCK inhibitors have been extensively applied in a variety of pathological studies, including cancer, neurodegeneration, kidney failure, asthma, glaucoma, osteoporosis, erectile dysfunction, and insulin resistance (31). For example, the ROCK inhibitor fasudil is on the Japanese market for the treatment of cerebral ischemic diseases. Currently, fasudil has been applied in more than 124,000 cases (32). In the field of ophthalmology, some reports suggest that the selective ROCK inhibitor Y-27632 can promote cell scratch repair in animal (cynomolgus monkey) CECs (33-35). The ROCK inhibitor Y-39983 can be used to treat glaucoma and has entered clinical trials (36).

In this study, to the best of our knowledge, for the first time, we examined the effects of in vitro co-culture of gradient concentrations of the ROCK inhibitor TZV with primary and passaged HCECs to observe changes in cell density, cell morphology, cell junctions, and ionic transporter expression of HCECs and identified the optimal drug concentration and time course of action of TZV in in vitro cultured HCECs. After either primary HCECs or passaged HCECs were co-cultured with $4 \mu \mathrm{M} \mathrm{TZV}$ for $48 \mathrm{~h}$, corneal EndMT/EMT was inhibited, as evidenced by the significant upregulation of the expression of the ZO-1 and adhesion protein E-cadherin and the obvious downregulation of $\mathrm{N}$-cadherin expression. This phenomenon of simultaneous upregulation of E-cadherin expression and downregulation of $\mathrm{N}$-cadherin expression is known as the cadherin switch (12,37); additionally, the mRNA expression levels of the EndMT/EMT-inducing gene snail are downregulated. Furthermore, cells in EndMT/EMT can particularly express $\alpha$-SMA, which is often used as an EndMT marker in CECs (16). In this study, the expression levels of the EndMT marker protein $\alpha$-SMA (38) was downregulated in response to the action of TZV at the above mentioned drug concentration and time course of action; in addition, the cell density increased, the cell volume decreased, tight junctions between the cells were enhanced and became clear, the cell morphology became hexagonal or pentagonal, and the expression of cellular ionic transporter expression protein $\mathrm{Na}^{+} / \mathrm{K}^{+}$-ATPase increased.

In conclusion, the novel ROCK inhibitor, TZV ( $4 \mu \mathrm{M})$, was effective in improving the morphology, cell junctions, and ionic transporter expression of HCECs via inhibiting EndMT/EMT but had no effect on HCEC proliferation. In the above described experiments, we determined a method with which to block EndMT in vitro while enhancing the expansion of CECs. More importantly, we observed that the number of primary HCECs acquired from the treatment of donor corneal rings left from human corneal transplantation with type IV collagenase expanded 10 -fold. In addition, these primary cells exhibited significant sensitivity to TZV. Therefore, we can use these cells to engineer a functional corneal endothelium. Moreover, for the drug application of TZV, we are inclined to perform direct anterior chamber injection of the drugs at the optimal concentration to improve the quality of endothelial cells in the early stage of decompensation. These experiments require further study at the organismal level. 


\section{Acknowledgements}

All authors are grateful for the financial support from the National Natural Science Foundation of China (no. 81371065), and acknowledge the support given by the Zhongshan Ophthalmic Center, Sun Yat-sen University for using the country's key laboratory and thank the Eye Bank of Guangdong Province for providing the corneal limbi left from corneal transplantation surgery.

\section{References}

1. Mishima S: Clinical investigations on the corneal endothelium-XXXVIII Edward Jackson Memorial Lecture. Am J Ophthalmol 93: 1-29, 1982.

2. Wulle KG: Electron microscopy of the fetal development of the corneal endothelium and Descemet's membrane of the human eye. Invest Ophthalmol 11: 897-904, 1972.

3. Laing RA, Sanstrom MM, Berrospi AR and Leibowitz HM: Changes in the corneal endothelium as a function of age. Exp Eye Res 22: 587-594, 1976.

4. Singh JS, Haroldson TA and Patel SP: Characteristics of the low density corneal endothelial monolayer. Exp Eye Res 115: 239-245, 2013.

5. He Z, Campolmi N, Gain P, Ha Thi BM, Dumollard JM, Duband S, Peoc'h M, Piselli S, Garraud O and Thuret G: Revisited microanatomy of the corneal endothelial periphery: New evidence for continuous centripetal migration of endothelial cells in humans. Stem Cells 30: 2523-2534, 2012.

6. Matsuda M, Sawa M, Edelhauser HF, Bartels SP, Neufeld AH and Kenyon KR: Cellular migration and morphology in corneal endothelial wound repair. Invest Ophthalmol Vis Sci 26: 443-449, 1985

7. Edelhauser HF: The resiliency of the corneal endothelium to refractive and intraocular surgery. Cornea 19: 263-273, 2000

8. Tan DTH, Dart JKG, Holland EJ and Kinoshita S: Corneal transplantation. Lancet 379: 1749-1761, 2012.

9. Hirata-Tominaga K, Nakamura T, Okumura N, Kawasaki S, Kay EP, Barrandon Y, Koizumi N and Kinoshita S: Corneal endothelial cell fate is maintained by LGR5 through the regulation of hedgehog and Wnt pathway. Stem Cells 31: 1396-1407, 2013.

10. Peh GS, Toh KP, Wu FY, Tan DT and Mehta JS: Cultivation of human corneal endothelial cells isolated from paired donor corneas. PLoS One 6: e28310, 2011.

11. Okumura N, Kay EP, Nakahara M, Hamuro J, Kinoshita S and Koizumi N: Inhibition of TGF- $\beta$ signaling enables human corneal endothelial cell expansion in vitro for use in regenerative medicine. PLoS One 8: e58000, 2013.

12. Engelmann $\mathrm{K}$ and Friedl P: Optimization of culture conditions for human corneal endothelial cells. In Vitro Cell Dev Biol 25: 1065-1072, 1989.

13. Zhu C and Joyce NC: Proliferative response of corneal endothelial cells from young and older donors. Invest Ophthalmol Vis Sci 45: 1743-1751, 2004.

14. Engelmann K, Bednarz J and Valtink M: Prospects for endothelial transplantation. Exp Eye Res 78: 573-578, 2004.

15. McNiven MA: Breaking away: Matrix remodeling from the leading edge. Trends Cell Biol 23: 16-21, 2013.

16. Roy O, Leclerc VB, Bourget JM, Thériault M and Proulx S: Understanding the process of corneal endothelial morphological change in vitro. Invest Ophthalmol Vis Sci 56: 1228-1237, 2015.

17. Lee JG, Ko MK and Kay EP: Endothelial mesenchymal transformation mediated by IL-1 $\beta$-induced FGF-2 in corneal endothelial cells. Exp Eye Res 95: 35-39, 2012.

18. Pipparelli A, Arsenijevic Y, Thuret G, Gain P, Nicolas M and Majo F: ROCK inhibitor enhances adhesion and wound healing of human corneal endothelial cells. PLoS One 8: e62095, 2013.
19. Gu L, Gao Q, Ni L, Wang M and Shen F: Fasudil inhibits epithelial-myofibroblast transdifferentiation of human renal tubular epithelial HK-2 cells induced by high glucose. Chem Pharm Bull 61: 688-694, 2013.

20. Park S, Kim D, Jung YG and Roh S: Thiazovivin, a Rho kinase inhibitor, improves stemness maintenance of embryo-derived stem-like cells under chemically defined culture conditions in cattle. Anim Reprod Sci 161: 47-57, 2015.

21. Yang H, Liu Z, Ma Y, Zhong C, Yin Q, Zhou C, Shi L, Cai Y, Zhao $\mathrm{H}$, Wang $\mathrm{H}$, et al: Generation of haploid embryonic stem cells from Macaca fascicularis monkey parthenotes. Cell Res 23: $1187-1200,2013$.

22. Tanaka T, Nishimura D, Wu RC, Amano M, Iso T, Kedes L, Nishida H, Kaibuchi K and Hamamori Y: Nuclear Rho kinase, ROCK2, targets p300 acetyltransferase. J Biol Chem 281: $15320-15329,2006$.

23. Lamouille S, Xu J and Derynck R: Molecular mechanisms of epithelial-mesenchymal transition. Nat Rev Mol Cell Biol 15: 178-196, 2014.

24. Huang RY, Guilford P and Thiery JP: Early events in cell adhesion and polarity during epithelial-mesenchymal transition. J Cell Sci 125: 4417-4422, 2012.

25. Kaibuchi K, Kuroda S and Amano M: Regulation of the cytoskeleton and cell adhesion by the Rho family GTPases in mammalian cells. Annu Rev Biochem 68: 459-486, 1999.

26. Somlyo AP and Somlyo AV: Signal transduction by G-proteins, rho-kinase and protein phosphatase to smooth muscle and non-muscle myosin II. J Physiol 522: 177-185, 2000.

27. Leung T, Chen XQ, Manser E and Lim L: The p160 RhoA-binding kinase ROK alpha is a member of a kinase family and is involved in the reorganization of the cytoskeleton. Mol Cell Biol 16: 5313-5327, 1996.

28. Tominaga T and Barber DL: Na-H exchange acts downstream of RhoA to regulate integrin-induced cell adhesion and spreading. Mol Biol Cell 9: 2287-2303, 1998.

29. Olson MF, Ashworth A and Hall A: An essential role for Rho, Rac, and Cdc42 GTPases in cell cycle progression through G1. Science 269: 1270-1272, 1995.

30. Zhang L, Valdez JM, Zhang B, Wei L, Chang J and Xin L: ROCK inhibitor Y-27632 suppresses dissociation-induced apoptosis of murine prostate stem/progenitor cells and increases their cloning efficiency. PLoS One 6: e18271, 2011.

31. Olson MF: Applications for ROCK kinase inhibition. Curr Opin Cell Biol 20: 242-248, 2008.

32. Liao JK, Seto M and Noma K: Rho kinase (ROCK) inhibitors. J Cardiovasc Pharmacol 50: 17-24, 2007.

33. Okumura N, Ueno M, Koizumi N, Sakamoto Y, Hirata K, Hamuro J and Kinoshita S: Enhancement on primate corneal endothelial cell survival in vitro by a ROCK inhibitor. Invest Ophthalmol Vis Sci 50: 3680-3687, 2009.

34. Okumura N, Koizumi N, Ueno M, Sakamoto Y, Takahashi H, Hirata K, Torii R, Hamuro J and Kinoshita S: Enhancement of corneal endothelium wound healing by Rho-associated kinase (ROCK) inhibitor eye drops. Br J Ophthalmol 95: 1006-1009, 2011.

35. Okumura N, Koizumi N, Kay EP, Ueno M, Sakamoto Y, Nakamura S, Hamuro J and Kinoshita S: The ROCK inhibitor eye drop accelerates corneal endothelium wound healing. Invest Ophthalmol Vis Sci 54: 2493-2502, 2013.

36. Tokushige H, Inatani M, Nemoto S, Sakaki H, Katayama K, Uehata $\mathrm{M}$ and Tanihara $\mathrm{H}$ : Effects of topical administration of y-39983, a selective rho-associated protein kinase inhibitor, on ocular tissues in rabbits and monkeys. Invest Ophthalmol Vis Sci 48: 3216-3222, 2007.

37. Yilmaz M and Christofori G: EMT, the cytoskeleton, and cancer cell invasion. Cancer Metastasis Rev 28: 15-33, 2009.

38. Piera-Velazquez S and Jimenez SA: Molecular mechanisms of endothelial to mesenchymal cell transition (EndoMT) in experimentally induced fibrotic diseases. Fibrogenesis Tissue Repair 5 (Suppl 1): S7, 2012. 